\title{
x
}

\section{Statistical Qualitative Analysis on Chemical Mechanical Polishing Process and Equipment Characterization}

\author{
Sang Jeen Hong ${ }^{\dagger}$, Jong Ha Hwang, and Dong-Sun Seo \\ Department of Electronic Engineering, Myongji University, Yongin 449-728, Korea
}

Received February 1, 2011; Revised March 7, 2011; Accepted March 11, 2011

\begin{abstract}
The characterization of the chemical mechanical polishing (CMP) process for undensified phophosilicate glass (PSG) film is reported using design of experiments (DOE). DOE has been used by experimenters to understand the relationship between the input variables and responses of interest in a simple and efficient way, and it typically is beneficial for determining the appropriatesize of experiments with multiple process variables and making statistical inferences for the responses of interest. The equipment controllable parameters used to operate the machine consist of the down force of the wafer carrier, pressure on the back side wafer, table and spindle speeds (SS), slurry flow (SF) rate, pad condition, etc. None of these are independent ofeach other and, thus, the interaction between the parameters also needs to be understoodfor improved process characterization in CMP. In this study, we selected the five controllable equipment parameters the most recommendedby process engineers, viz. the down force (DF), back pressure (BP), table speed (TS), SS, and SF, for the characterization of the CMP process with respect to the material removal rate and film uniformity in percentage terms. The polished material is undensified PSG which is widely used for the plananization of multi-layered metal interconnects. By statistical modeling and the analysis of the metrology data acquired from a series of $2^{5-1}$ fractional factorial designs with two center points, we showed that the DF, BP and TS have the greatest effect on both the removal rate and film uniformity, as expected. It is revealed that the film uniformity of the polished PSG film contains two and three-way interactions. Therefore, one can easily infer that process control based on a better understanding of the process is the key to success in current semiconductor manufacturing, in which the size of the wafer is approaching $300 \mathrm{~mm}$ and is scheduled to continuously increase up to $450 \mathrm{~mm}$ in or slightly after 2012 .
\end{abstract}

Keywords: Phophosilicate glass, Chemical mechanical polishing, Design of experiment

\section{INTRODUCTION}

With the continuous scaling down of device technology and complicated integration of multi-layered integrated circuits (ICs), planarization at each level of the inter-layer dielectric has become more significant in current system IC manufacturing. The level of complexity for system IC integration, such as microprocessors and digital signal processors, in general, is more complex than that of memory devices. In the era of mid-size integration, which may contain less than a hundred thousand devices, planarization was not a required process step in IC fabrication. However, the need for aplanar surface for improving the

\footnotetext{
${ }^{\dagger}$ Author to whom all correspondence should be addressed: E-mail: samhong@mju.ac.kr
}

depth of focus and photo-patterning resolution in multi-level IC integration requires each level of the surface to be planar, and this planarization has become a critical step in terms of process integration [1].

For the planarization of the wafer surface, numerous methods has been developed, such as doped glass reflow, hydrophobicity, spin etch planarization, spin on deposition, reactive ion etch etch-back, spin on deposition etch back, and chemical mechanical polishing (CMP) [2]. CMP is a widely used planarization method for global planarization in the semiconductor industry, because it provides compatability with various materials ranging from dielectrics to metals. It is also useful for the planarization of multi-material surfaces and reduction of the severe topography.

Although CMP, compared to other suggested planarization methods, provides increased throughput in manufacturing, the 
Table 1. Factors and their ranges in the experiment.

\begin{tabular}{lllll}
\hline Factor & Units & + & 0 & - \\
\hline \hline Down force & psi & 15 & 11 & 6 \\
Back pressure & Psi & 4 & 2.5 & 1 \\
Table speed & $\mathrm{rpm}$ & 100 & 80 & 60 \\
Spindle speed & $\mathrm{rpm}$ & 40 & 25 & 10 \\
Slurry flow & $\mathrm{mL} / \mathrm{min}$ & 150 & 100 & 50 \\
\hline
\end{tabular}

control of the process becomes more complicated with increasing device integration density and the introduction of newer materials, which significantly narrows the process latitude with the development of device fabrication technology. The need to control the process parameters to achieve certain levels of process yield cannot be over-emphasized. There are a large number of tool or process parameters, but they can be grouped into the following three classes: 1) tool operating related, such as the pressure, velocity, and temperature; 2) slurry related, such as abrasive, slurry and fluid; and 3) pad related.

The separate consideration of each group of parameters for the analysis of the process may not justifiable since it is not independent with respect to the results or outputs of interest, but the correlation and interaction with each other to some degree. In this paper, we employed the statistical design of experiments (DOE) to investigate the statistical inference of the individual parameters and their interactions for the figure of merit of the CMP process, removal rate and uniformity. Employing a fractional factorial design with five process parameters, a series of CMP experiments was followed by the DOE, and the measured wafer metrology was statistically modeled and analyzed to draw objective conclusions.

\section{EXPERIMENTS}

\subsection{Material and process}

The data employed for the statistical analysis were acquired from a set of CMPs processes of phosphosilicate glass (PSG) that are frequently used in the back end of line process in system IC integration. PSG not only provides a lower dielectric contact over silicon oxide, but also reduces the depth of the steps created by metallization.

The thickness of the PSG thin film, as deposited on prime grade $p$-type $<100>$ silicon wafers, was firstly measured, and the differences in the thicknesses of the film were utilized to determine the uniformity as well as the removal rate. The measurement of the film thickness was performed using nanospec, and the thickness of five different locations, viz. the center, top, bottom, left and right, was measured to calculate the Among the numerous tool parameters, five controllable parameters were included as the input factors in this experiment, and their name and range of process recipes are presented in Table 1. While a series of designed experiment was performed, other factors, such as the types of pad and slurry, table temperature, and pad condition, were fixed. A schematic illustration of the tool operation with the operating parameters ispresented in Fig. 1.

\subsection{Design of experiment}

DOE is a statistically driven experimental design method which provides the systematical determination of experiment and enables statistical analysis to be conducted via analysis of variance (ANOVA) and regression modeling. It also allows the main effects of the parameters employed and interactions be-

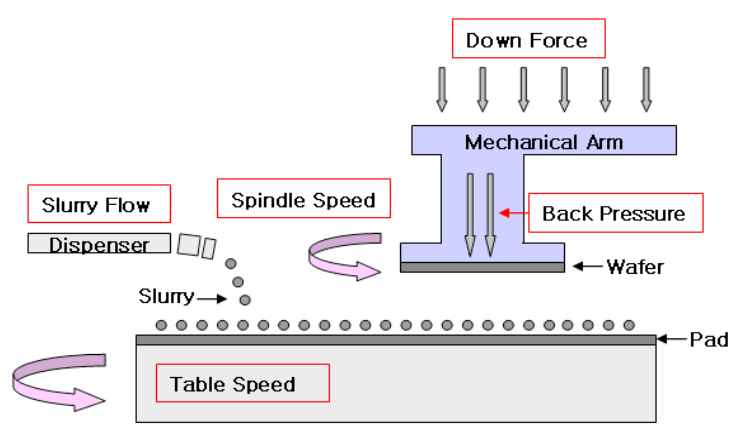

Fig. 1. A schematic of chemical mechanical polishing tool and process.

tween the parameters to be visualized for the given responses of interest, herein the removal rate and calculated percent uniformity.

The benefit of DOE also extends to the maximization of the data analysis with the least number of experiments. If a process has more than just a few steps whose possible values have a large range, the number of experiments needed for process characterization can be prohibitively large. In addition, the role of each step in determining the final outcome is generally not clear. The traditional method of collecting large quantities of data, by holding each factor constant in turn until all possibilities have been tested, is an approach that quickly becomes impossible as the number of factors increases. Statistical experimental design is a systematic and efficient alternative methodology for characterization and modeling using a relatively small number of experiments. The statistical data analysis was performed by investigating the DOE data from a $2^{5-1}$ fractional factorial design with multiple center points.

A randomized experimental run order was strictly followed in order to avoid any experimenter bias or tool condition associated with time. Two responses of interest, the removal rate $(R R)$ and percent non-uniformity $(N U)$, were calculated using the simple equation below:

$$
\begin{aligned}
& R R(\AA / \mathrm{min}) \\
& =\frac{\sum_{i=1}^{n}(\text { Initial film thickness }- \text { Fianl filmthickness }) / n}{\text { Time }} \\
& N U(\%)=\frac{\text { Range of final thickness }}{2 \times \text { Average of final thickness }} \times 100(\%)
\end{aligned}
$$

While the statistical analysis of a given data set, we found that the percent non-uniformity does not follow a normal distribution and, thus, a Box-Cox transformation was initially taken before any further statistical data analysis, which will be described in detail in the following section.

\section{REGRESSION MODELING}

\subsection{Statistical assumption}

A key underlying assumption in statistics is that the given data follows anormal distribution, and checking the normality before any statistical analysis is highly recommended. Otherwise, misleading conclusions may be drawn. We checked the data normality for both responses, and the percent non-uniformity 
Normal Probability Plot of the Residuals

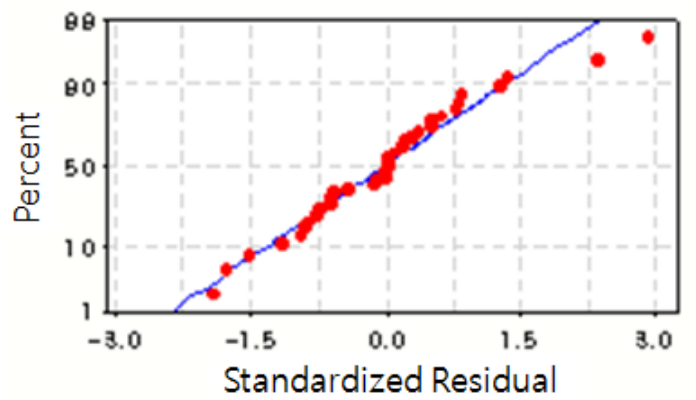

Histogram of the Residuals

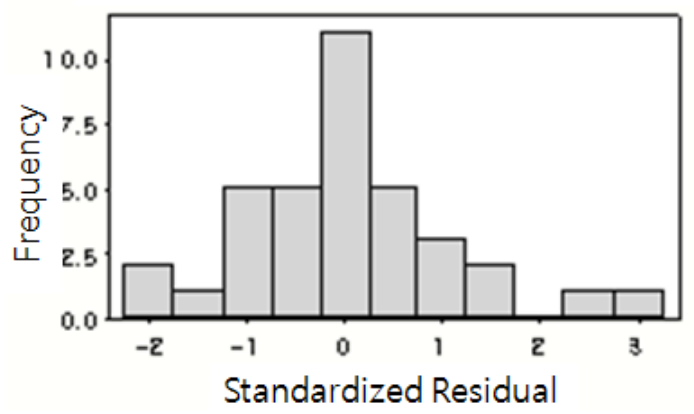

Residuals vs. Fitted Values

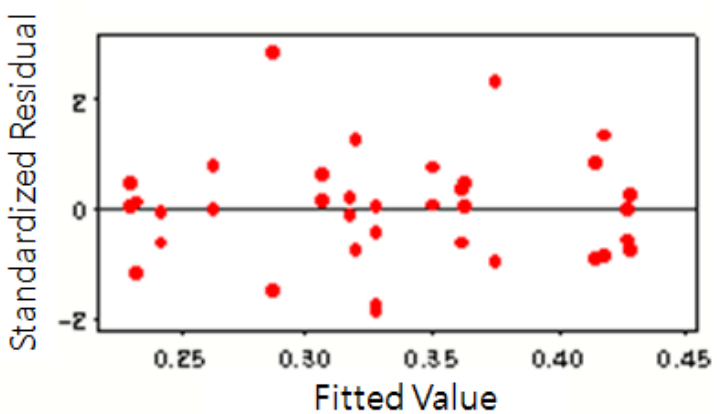

Residual vs. Run Order

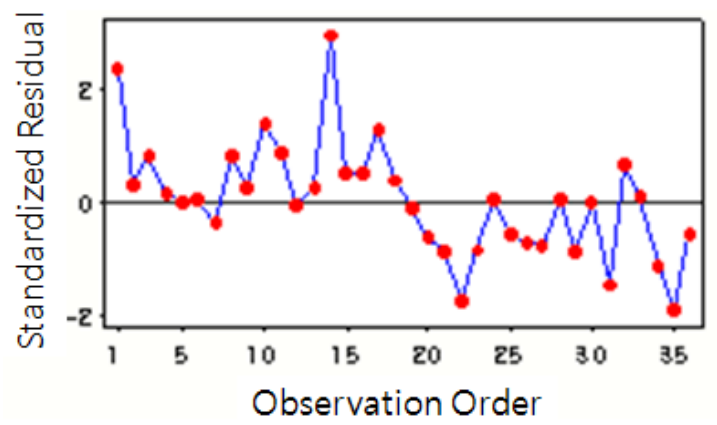

Fig. 2. Residual plots for NU_BCT.

$(N U)$ showed a relatively low degree of normality. The central limit theorem can be used for the justification of the normality with limited number of experiments, but this may jeopardize the statistically driven conclusion. Thus, we employed the Box-Cox transformation with $\lambda=-0.5$ for the acquired $N U$ data [3].

The transformed $N U$ data, hereafter referred to as $N U \_B C T$, was then used for regression modeling, and the graphical result of the residual analysis on the transformed data set is provided in Fig. 2. From the first figure, it can be seen that the transformed data follows a normal distribution and the residuals are randomly distributed in the rest of the figure. Therefore, it is clear

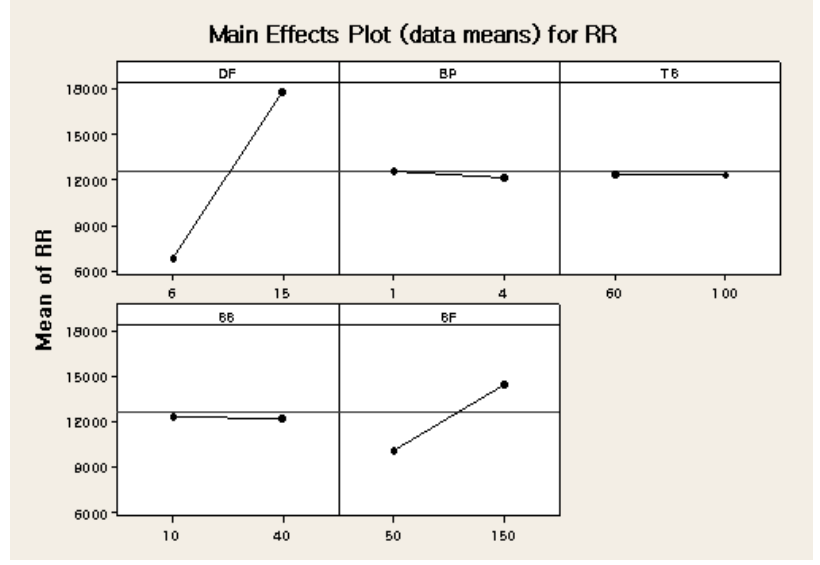

Fig. 3. Main effects plot (data mean) for removal rate (RR).

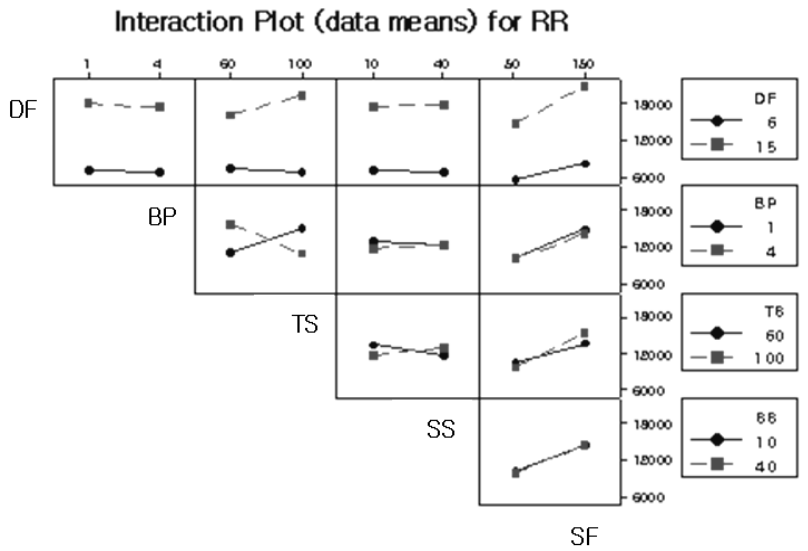

Fig. 4. Interaction plot (data mean) for removal rate (RR).

that the transformation of the NU data is necessary for further analysis.

\subsection{Removal rate}

Once the statistical requirement for further analysis was satisfied, we performed a qualitative analysis for the purpose of understanding the relationships between the parameters. Statistical analysis and regression modeling certainly provide a great amount of specific information, but sufficient background knowledge of statistics is required to interpret the result However, this paper focuses on qualitative analysis to provide insight into first level equipment engineers in their operation.

At first glance, the factors having the most influence are the down force (DF) and slurry flow (SF), and it is therefore not surprising that the mechanical polishing mechanism is dominant for phosphosilicate glass. The initially considered variables, viz. the back pressure (BS), spindle speed (SS), and table speed (TS), were found to be insignificant factors from the main effect plot, as shown in Fig. 3. The interactions of BP-TS and TS-SS with respect to RR are shown in Fig. 4, but they are among the insignificant parameters. On the other hand, the BP can affect the removal rate with $\mathrm{DF}$, and it has similar downward forcing mechanisms on the wafer in this process. The qualitative analysis of the two types of plots allows engineers to consider the three most important tool parameters to keep constant in the device manufacturing process. One can conduct a qualitative analysis, such as ANOVA analysis and regression modeling, to achieve the 


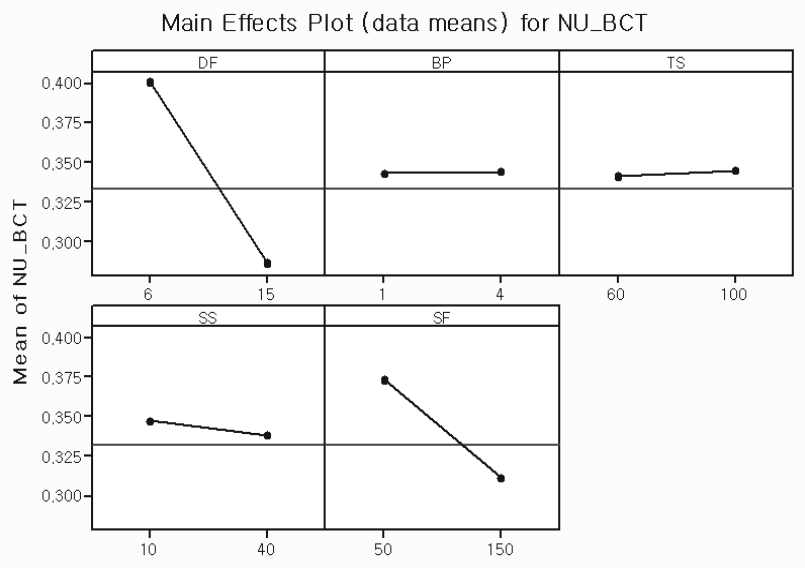

Fig. 5. Main effects plot (data means) for NU_BCT.

degree of control required for this specific process, but numerical analysis is outside of the scope of the work. The regression equation derived for the removal rate is as follows.

$$
R R=182.70+227.47 \cdot D F+1.89 \cdot B F+5.70 \cdot D F \cdot B P-3.66 \cdot T S \cdot S S
$$

\subsection{Percent non-uniformity}

From the main effect plot in Fig. 5, only two parameters, DF and SF, are shown to have a statistically significant effect on the non-uniformity, but some interactions between the other parameters also obviously exist. The qualitative analysis on the percent non-uniformity shows similar results for the removal rate, because its calculation includes the average value of the removal rate from a different location.

For a better understanding of the quantitative information, stepwise regression modeling was carried out. Initially, we included two-way interactions, and a very poor model fit was obtained with $R$-sq values as high as $40 \%$. Including the three-way interaction terms increased the model fit to almost $60 \%$, but the statistical modeling still retained some degree of residual errors from the modeling. Providing the built regression equation and ANOVA table would give much more detailed information for the analysis, but the qualitative analysis still conceptually holds when the model fit does not provide a good value of R-sq.

$N U=-0.71+0.91 \cdot D F+1.29 \cdot B F-0.54 \cdot S F-0.23 \cdot D F \cdot B P+0.01 \cdot D F \cdot S F$
The statistical modeling might not be as good as expected, but we found that the DF and SF are the most significant factors in CMP. In addition, the wafer back pressure interacts with the down force to cause a change of the removal rate, and the spindle speed can interact with the SF in terms of the CMP uniformity. For successful semiconductor manufacturing, these tool parameters should be tightly controlled for the purpose of increasing the process yield.

\section{CONCLUSIONS}

Five parameters associated with process characterization were investigated, and their effects on the removal rate and non-uniformity were determined from the regression equation generated from the regression analysis. From the analysis, it was found that the main effects of down force, back pressure, and table speed, and two- and three-way interaction terms, have a significant effect in the analysis. Ultimately, the non-uniformity is a complex response resulting from the high degree of interactions of more than two parameters, and the control of the non-uniformity in larger wafer fabrication processes is very valuable, especially for high volume manufacturing environments. The removal rate of the CMP process is mainly affected by the down force and spindle speed, but there obviously exists some degree of interaction that cannot be ignored.

\section{ACKNOWLEDGMENTS}

This research was supported by the Semiconductor Progress Diagnosis Research Center at Myongji University, and the authors are grateful to the members of the Intelligent Micro-Nano Process Fab at Myongji University for their technical supports.

\section{REFERENCES}

[1] S. Wolf and R. N. Tauber, Silicon Processing for the VLSI Era, 2nd ed. (Lattice Press, Sunset Beach, 2002).

[2] G. E. P. Box, W. G. Hunter, and J. S. Hunter, Statistics for Experimenters: An Introduction to Design, Data Analysis, and Model Building (Wiley, New York, 1978).

[3] P. Y. Chen, C. J. Wu, C. N. Ko, and J. T. Jeng, Artif. Life Robot. 14, 62 (2009) [DOI: 10.1007/s10015-009-0629-6]. 